\title{
PREVALENCIA DEL JUEGO PATOLÓGICO EN EL ALCOHOLISMO: UN ESTUDIO EXPLORATORIO
}

\author{
JAVIER FERNÁNDEZ-MONTALVO ${ }^{1}$ \\ NATALIA LANDA ${ }^{2}$ \\ JOSÉ J. LÓPEZ-GOÑI ${ }^{3}$ \\ ${ }^{1}$ Universidad Pública de Navarra \\ ${ }^{2}$ Centro de Salud Mental de Tudela \\ ${ }^{3}$ Fundación Proyecto Hombre \\ (Aceptado en diciembre de 2004)
}

\begin{abstract}
En este estudio se lleva a cabo un análisis de la prevalencia del juego patológico en $\mathbf{5 0}$ alcohólicos que acuden en busca de tratamiento. Para ello, se utilizaron los criterios diagnósticos del DSM-IV-TR para el juego patológico y el Cuestionario de Juego Patológico de South Oaks (SOGS). Los resultados obtenidos mostraron que el $20 \%$ de los alcohólicos de la muestra presentaba un diagnóstico comórbido de ludopatía. Además, un $12 \%$ adicional obtenía una puntuación en el SOGS indicadora de juego problemático. En suma, el $32 \%$ de la muestra presentaba síntomas de juego clínicamente significativos. La comparación entre los alcohólicos con y sin ludopatía asociada mostró diferencias significativas en las variables relacionadas con el consumo de alcohol, que eran más altas en la submuestra de alcohólicos ludópatas, pero no en la sintomatología psicopatológica evaluada con el SCL-90-R. Se comentan las implicaciones de este estudio para la práctica clínica y la investigación futura.
\end{abstract}

Palabras-clave: Juego patológico, alcoholismo, comorbilidad, evaluación.

\section{Prevalence of pathological gambling in the alcoholism: An exploratory study}

In this paper, the prevalence of pathological gambling in 50 treatment-seeking alcoholics was estimated. The DSM-IV-TR diagnosis criteria for pathological gambling and the Spanish version of the South Oaks Gambling Screen (SOGS) were used. Results showed a rate of $20 \%$ of alcoholics with a comorbid diagnosis of pathological gambling. Furthermore, an additional $12 \%$ of the alcoholic sample had relevant symptoms for problem gambling. In sum, the $32 \%$ of the sample reported clinically significant gambling-related symptoms. Comparison between alcoholics with and without pathological gambling showed significant differences in alcohol variables, which were higher in gambling sample. However there were no significant differences between groups in the psychopathological symptoms assessed with the SCL-90-R. Implications of these results for further research and clinical practice are commented upon.

Key words: Pathological gambling, alcoholism, comorbidity, assessment.

\section{INTRODUCCIÓN}

En la sociedad actual, los trastornos adictivos en general, y el alcoholismo en particular, constituyen un fenómeno psicopatológico de gran relevancia clínica y social. Por lo que se refiere específica-

Correspondencia: Javier Fernández-Montalvo, Universidad Pública de Navarra, Campus de Arrosadía, 31006 Pamplona. Correo-e: fernandez.montalvo@unavarra.es mente al alcoholismo, las tasas de prevalencia tan elevadas que se observan en la población, así como el descenso tan importante que se ha producido en la edad de los pacientes aquejados de una dependencia alcohólica (Fernández-Montalvo, Landa, López-Goñi, Lorea y Zarzuela, 2002), se relacionan, probablemente, con la facilidad de acceso al alcohol, con su integración cultural y con su carácter legal. 
Algo similar está ocurriendo en el ámbito de las adicciones sin drogas. La ludopatía, y dentro de ella, la dependencia de las máquinas tragaperras, constituye la adicción psicológica de mayor prevalencia en nuestros días (Echeburúa, 1999). Ello se relaciona también con la facilidad de acceso, con la integración cultural de este tipo concreto de juego y con las características específicas del funcionamiento de estas máquinas.

En cualquier caso, en los últimos años han sido varios los autores que han señalado los aspectos comunes y diferenciales entre las adicciones químicas - las clásicas adicciones- y las adicciones sin drogas - las denominadas como nuevas adicciones- (Alonso-Fernández, 1996; Echeburúa, 1999). En este sentido, una característica importante presente en las conductas adictivas es la elevada tasa de polidependencia -adicción simultánea a varias sustanciasque se observa en el ámbito clínico. De hecho, es muy poco frecuente encontrar pacientes aquejados de una dependencia a una única sustancia.

Asimismo, es destacable la elevada comorbilidad de las distintas conductas adictivas con otros cuadros clínicos. Cuando una persona es adicta a una sustancia, lo más habitual -hasta en un $50 \%-75 \%$ de los casos- es que presente otro trastorno adicional (Casas, 1994). Ello ha producido un interés creciente por el estudio de los trastornos duales en las conductas adictivas. En el caso concreto del alcoholismo, los trastornos duales más frecuentes son, por una parte, los trastornos de la personalidad (FernándezMontalvo y Landa, 2003); y por otra, la depresión - más en las mujeres-, los trastornos de ansiedad -en uno y otro sexo- y el abuso de otras drogas -más en los hombres- (Echeburúa, 2001).

Recientemente se ha puesto de manifiesto la frecuente coexistencia del alcoholismo con los trastornos del control de los impulsos. Así, por ejemplo, en el estudio de Lejoyeux, Feuche, Loi, Solomon y Ades (1999), con una muestra de 79 alcohólicos, el 38\% cumplía los criterios diagnósticos para un trastorno del control de los impulsos. Entre todos ellos destacaba por su mayor frecuencia el juego patológico. Asimismo, son varios los estudios que muestran mayores tasas de juego patológico entre la población drogodependiente en general (Spunt, Dupont, Lesieur, Liberty y Hunt, 1998; Toneatto y Brennan, 2002; Toneatto, Ferguson y Brennan, 2003), así como su influencia en los resultados terapéuticos obtenidos (Ledgerwood y Downey, 2002).

Son todavía escasos los estudios llevados a cabo sobre la prevalencia del juego patológico entre la población alcohólica. Sin embargo, los resultados encontrados hasta la fecha muestran una comorbilidad alta entre ambas adicciones, con tasas de juego patológico que afectan a entre el $8 \%$ y el $33 \%$ de los alcohólicos (Daghestani, Elenz y Crayton, 1996; Lesieur, Blume y Zoppa, 1986; Rodriguez-Martos, 1989; Sellman, Adamson, Robertson, Sullivan y Coverdale, 2002; Welte, Barnes, Wieczorek y Tidwell, 2004). La prevalencia del juego patológico en la población general es aproximadamente del 2\% (Becoña, 1996; Bland, Newman, Orn y Stebelsky, 1993; Ladouceur, 1991; Volberg y Abbot, 1994; Volberg y Steadman, 1988, 1989). Ello pone de manifiesto la necesidad de evaluar el juego patológico entre los alcohólicos que acuden en busca de tratamiento. No se debe olvidar, en este sentido, que el tratamiento de cualquier conducta adictiva puede generar una dependencia sustitutiva y aumentar la implicación del sujeto en otra adicción (Daghestani et al., 1996; Rodríguez-Martos, 1989).

Desde la perspectiva contraria, también es frecuente encontrar altas tasas de abuso de alcohol y de alcoholismo entre los jugadores. Los estudios llevados a 
cabo hasta la fecha arrojan cifras que oscilan entre el $11 \%$ y el $70 \%$, con una amplia variabilidad entre los distintos estudios (Becoña, 1993; Bland et al., 1993; Ciarrocchi y Richardson, 1989; Dell, Ruzicka y Palisi, 1981; Kausch, 2003; Martínez-Pina, Guirao de Parga, Fusté, Serrat, Martín y Moreno, 1991; Ramírez, McCormick, Russo y Taber, 1983; Specker, Carlson, Edmonson, Johnson y Marcotte, 1996).

El objetivo principal de este estudio es llevar a cabo una evaluación de los problemas de juego patológico presentes en una muestra de 50 pacientes alcohólicos que acuden en busca de tratamiento. Se trata, en primer lugar, de establecer la tasa de prevalencia de la ludopatía entre los alcohólicos de la muestra y, en segundo lugar, de comparar la gravedad de la dependencia alcohólica en función de la existencia de un diagnóstico asociado de juego patológico.

\section{MÉTODO}

\section{Participantes}

La muestra de este estudio está compuesta por 50 alcohólicos que acudieron en busca de ayuda terapéutica al programa de tratamiento ambulatorio del alcoholismo de Proyecto Hombre de Navarra, durante el período comprendido entre noviembre de 2003 y agosto de 2004. De acuerdo con los criterios de admisión al estudio, los paciente tenían que: a) cumplir los criterios diagnósticos del DSMIV-TR (American Psychiatric Association, 2000) para la dependencia alcohólica; b) tener una puntuación igual o superior a 11 en la versión española del Müncher Alkoholismus Test (MALT) (Feuerlein, Ringer, y Kufner, 1977); c) tener una edad comprendida entre los 18 y 65 años; y d) dar su consentimiento informado para participar en el estudio.
Por lo que se refiere a las principales características sociodemográficas de la muestra seleccionada de alcohólicos, la edad media era de 43,8 años $(D T=10,5)$, 38 sujetos $(76 \%)$ eran hombres y 12 (24\%) mujeres. Todo ellos pertenecían a un nivel socioeconómico de clase media y media-baja.

\section{Medidas de evaluación}

El Müncher Alkoholismus Test (MALT) (Feuerlein et al. 1977) es una prueba diagnóstica que está compuesta por dos partes. La primera (MALT-O), de carácter objetivo, consta de 7 ítems que son cumplimentados por el terapeuta a partir de los datos procedentes de la exploración clínica, del historial del paciente y de los análisis de laboratorio. Cada respuesta positiva recibe una puntuación de 4 puntos. La segunda parte del instrumento (MALT-S), de carácter subjetivo, consta de 27 ítems auto-administrados, con un formato de respuesta dicotómico $(0=$ no; 1 = sí). Una puntuación total en el instrumento igual o superior a 11, el punto de corte, sugiere la presencia de un diagnóstico de alcoholismo. En esta investigación se ha utilizado la versión española de Rodríguez-Martos y Suárez (1984).

El Cuestionario de Juego Patológico de South Oaks (SOGS) (Lesieur y Blume, 1987; validación española de Echeburúa, Báez, Fernández-Montalvo y Páez, 1994) es un cuestionario de 20 ítems, elaborado con arreglo a los criterios del DSM-III (APA, 1980), pero que se adapta también a los del DSM-IV-TR (APA, 2000). En la versión española una puntuación igual o superior a 4 indica la presencia del juego patológico. Además, la puntuación obtenida en este cuestionario constituye un reflejo de la gravedad de la adicción al juego. Un análisis más detallado de las propiedades psicométricas del SOGS puede encontrarse en Echeburúa et al. (1994). 
El SCL-90-R (Derogatis, 1992; versión española de González de Rivera, 2002) es un cuestionario autoadministrado de evaluación psicopatológica general. Consta de 90 ítems, con 5 alternativas de respuesta en una escala de tipo Likert, que oscilan entre 0 (nada) y 4 (mucho). El cuestionario tiene como objetivo reflejar los síntomas de malestar psicológico de un sujeto. Al haberse mostrado sensible al cambio terapéutico, se puede utilizar tanto en una única evaluación como en evaluaciones repetidas. El SCL-90-R está constituido por nueve dimensiones de síntomas primarios (somatización, obsesión-compulsión, sensibilidad interpersonal, depresión, ansiedad, hostilidad, ansiedad fóbica, ideación paranoide y psicoticismo). Además, ofrece tres índices globales que reflejan el nivel de gravedad del sujeto.

\section{Procedimiento}

Una vez seleccionados los sujetos de la muestra de acuerdo con los criterios previamente indicados, la evaluación de los alcohólicos se llevó a cabo en el marco de la evaluación pretratamiento y constó de dos sesiones. En la primera se recogieron los datos relacionados con las características sociodemográficas y con el consumo de alcohol. En la segunda sesión se evaluó la presencia de sintomatología psicopatológica (con el SCL-90-R) y de un diagnóstico comórbido de juego patológico (con los criterios del DSMIV-TR y con el SOGS).

\section{RESULTADOS}

Para determinar las características de la muestra, la sintomatología asociada y la tasa de comorbilidad entre el alcoholismo y el juego patológico se han utilizado análisis estadísticos de carácter des- criptivo (porcentajes, medias y desviaciones típicas). Asimismo, la comparación entre los grupos de alcohólicos con y sin ludopatía asociada se ha llevado a cabo mediante la prueba de Chi cuadrado, en el caso de las variables categóricas, y la U de Mann-Whitney, en el caso de las variables cuantitativas.

\section{Características del consumo de alcohol}

Por lo que se refiere al consumo de alcohol, la puntuación media obtenida en el MALT es de 29,5 (DT =8,2), con un rango que oscila entre 11 y 50 puntos. El consumo de alcohol se caracteriza por ser frecuente (6-7 días a la semana), con una media de consumo de 216,7 gramos diarios $(D T=123,6$, rango $=50-640$ gramos/día). Además, los pacientes presentan una antigüedad media de la dependencia alcohólica de 12 años.

\section{Prevalencia del juego patológico}

El 20\% (10 sujetos) de los alcohólicos de la muestra cumple los criterios diagnósticos del DSM-IV-TR (APA, 2000) para el juego patológico. Esta tasa de comorbilidad coincide totalmente con la evaluación llevada a cabo con el SOGS.

El punto de corte de la versión española del SOGS es de 4 puntos. La puntuación media obtenida por la muestra total de alcohólicos es de 1,85 puntos $(D T=2,96)$. Sin embargo, desde una perspectiva clínica, la tasa de alcohólicos con una puntuación igual o superior a 4 es de 10 sujetos (el $20 \%$ de la muestra total). Además, un $12 \%$ adicional obtiene 2 o 3 puntos en el SOGS, considerado, en la versión española, como un indicador de juego problemático. Estos datos reflejan la existencia de un $32 \%$ de alcohólicos con problemas de juego clínicamente significativos $(20 \%$ con juego patológico y $12 \%$ con juego problemático). 
Comparación entre los dos grupos de alcohólicos

Desde una perspectiva sociodemográfica, la única diferencia significativa entre los jugadores patológicos y los no ludópatas es el sexo: el 100\% de los alcohólicos ludópatas son hombres, frente al $70 \%$ de los alcohólicos no ludópatas $\left(\chi^{2}=3,88 ; p<0,05\right)$.

Los resultados de la comparación entre los alcohólicos con y sin ludopatía asociada en las variables relacionadas con el consumo de alcohol se presentan en la Tabla 1.

Como se puede observar en la Tabla, existen diferencias estadísticamente sig- nificativas en tres variables: la frecuencia de consumo, la puntuación en el MALT y la presencia de otras dependencias. En concreto, los alcohólicos con un diagnóstico asociado de juego patológico presentan una mayor frecuencia de consumo de alcohol (consumo diario en la mayor parte de los pacientes de este grupo), una mayor puntuación en el MALT (reflejo de una mayor gravedad de la adicción al alcohol) y una mayor presencia de otras dependencias asociadas de forma simultánea (polidependencia).

Por lo que se refiere a la sintomatología psicopatológica asociada, no hay diferencias significativas entre ambos grupos.

Tabla 1. Comparación en las variables de consumo de alcohol

\begin{tabular}{|c|c|c|c|c|}
\hline Variables & $\begin{array}{c}\text { Muestra total } \\
N=50 \\
\text { Media (DT) }\end{array}$ & $\begin{array}{c}\text { Con juego patológico } \\
N=10 \\
\text { Media }(D T)\end{array}$ & $\begin{array}{c}\text { Sin juego patológico } \\
N=40 \\
\text { Media }(D T)\end{array}$ & $U$ \\
\hline \multicolumn{5}{|l|}{ MALT } \\
\hline MALT-Subjetivo & $18,7(3,8)$ & $21,1(2,3)$ & $18,3(3,9)$ & 101,1 * \\
\hline MALT-Objetivo & $10,5(5,5)$ & $12,4(4,7)$ & $10,3(5,7)$ & 130,5 \\
\hline MALT-Total & $29,5(8,2)$ & $33,5(6,5)$ & $28,9(8,4)$ & $106,5^{\star}$ \\
\hline Años de dependencia & $11,8(6,8)$ & $13,1(7,2)$ & $11,2(6,9)$ & 155,5 \\
\hline Gramos diarios & $216,7(123,6)$ & $248,1(130,8)$ & $216,4(123,4)$ & 157,5 \\
\hline Variables & $\begin{array}{c}\text { Muestra total } \\
N=50 \\
N(\%)\end{array}$ & $\begin{array}{c}\text { Con juego patológico } \\
N=10 \\
N(\%) \\
\end{array}$ & $\begin{array}{c}\text { Sin juego patológico } \\
N=10 \\
N(\%)\end{array}$ & $x^{2}$ \\
\hline \multicolumn{5}{|l|}{ Patrón de consumo } \\
\hline $\begin{array}{l}\text { Continuo } \\
\text { Episódico } \\
\end{array}$ & $\begin{array}{l}39(78 \%) \\
11(22 \%) \\
\end{array}$ & $10(100 \%)$ & $\begin{array}{l}29(72,5 \%) \\
11(27,5 \%) \\
\end{array}$ & 3,5 \\
\hline \multicolumn{5}{|l|}{ Frecuencia de consumo } \\
\hline $\begin{array}{l}\text { Diario } \\
\text { 4-5 días/semana } \\
\text { 2-3 días/semana } \\
\end{array}$ & $\begin{array}{r}36(72 \%) \\
5(10 \%) \\
9(18 \%) \\
\end{array}$ & $10(100 \%)$ & $\begin{array}{rr}26 & (65 \%) \\
5 & (12,5 \%) \\
9 & (22,5 \%) \\
\end{array}$ & $4,9^{*}$ \\
\hline \multicolumn{5}{|l|}{ Tratamientos previos } \\
\hline $\begin{array}{l}\text { Ninguno } \\
\text { Uno } \\
\text { Dos } \\
\text { Tres o más }\end{array}$ & $\begin{array}{r}19(38 \%) \\
16(32 \%) \\
7(14 \%) \\
8(16 \%)\end{array}$ & $\begin{array}{l}4(40 \%) \\
4(40 \%) \\
1(10 \%) \\
1(10 \%)\end{array}$ & $\begin{array}{rr}15 & (37,5 \%) \\
12 & (30 \%) \\
6 & (15 \%) \\
7 & (17,5 \%)\end{array}$ & 0,02 \\
\hline \multicolumn{5}{|l|}{ Otras dependencias } \\
\hline $\begin{array}{l}\text { Sí } \\
\text { No }\end{array}$ & $\begin{array}{l}18(36 \%) \\
32(64 \%)\end{array}$ & $\begin{array}{l}7(70 \%) \\
3(30 \%)\end{array}$ & $\begin{array}{l}11(27,5 \%) \\
29(72,5 \%)\end{array}$ & 6,3 * \\
\hline
\end{tabular}

${ }^{\star} p<0,05$ 
Los resultados obtenidos en el SCL-90-R (Tabla 2) reflejan que ambos grupos de alcohólicos son homogéneos en estas variables.

Con el objetivo de comprobar si la ausencia de diferencias entre los dos subgrupos se debía, en realidad, a una ausencia de síntomas psicopatológicos relevantes en la muestra total de alcohólicos, se llevó a cabo una comparación en todas las dimensiones del SCL-90-R entre la muestra del estudio y la muestra normativa del instrumento. Los resultados pusieron de manifiesto que la puntuación de la muestra total de alcohólicos en todas las dimensiones del SCL-90-R era significativamente superior a la obtenida por la muestra normativa del instrumento $(p<0,05)$, excepto en el Índice de Distrés de Síntomas Positivos.

\section{DISCUSIÓN}

En este estudio se ha llevado a cabo una evaluación de los problemas de juego patológico presentes en una muestra de pacientes alcohólicos. Los resultados encontrados apoyan la creciente evidencia empírica que muestra tasas de prevalencia elevadas de juego patológico entre la población alcohólica. Al igual que se ha obtenido en otros estudios previos (Daghestani et al., 1996; Lesieur et al., 1986; Rodriguez-Martos, 1989; Sellman et al., 2002; Welte et al., 2004), el juego patológico tiene una presencia más alta entre los alcohólicos que entre la población normal (en torno al $2 \%$ en los diferentes estudios epidemiológicos).

Más en concreto, los resultados de este estudio muestran que el $20 \%$ de los alcohólicos de la muestra cumplen los criterios diagnósticos del DSM-IV-TR para el juego patológico, a pesar de no haber consultado por ello. Además, se ha detectado la presencia de un $12 \%$ adicional de alcohólicos que obtienen una puntuación en el SOGS indicativa de un posible juego problemático. Estos datos muestran que el $32 \%$ de la muestra presenta problemas de juego clínicamente significativos.

Esta cifra tan elevada es preocupante, ya que los programas habituales de inter-

Tabla 2. Comparación en el SCL-90-R

\begin{tabular}{lcrrr}
\hline \multicolumn{1}{c}{ Variables } & Muestra total & Con juego patológico & Sin juego patológico \\
& Media $(D T)$ & $\begin{array}{c}N=10 \\
\text { Media }(D T)\end{array}$ & $\begin{array}{c}N=40 \\
\text { Media }(D T)\end{array}$ & $U$ \\
\hline Índice Global de Gravedad & $0,89(0,71)$ & $1,01(0,7)$ & $0,9(0,7)$ & 148 \\
Índice de Distrés de Síntomas Positivos & $1,6(0,59)$ & $1,7(0,4)$ & $1,7(0,6)$ & 146 \\
Total de Síntomas Positivos & $41,5(22,4)$ & $49,3(24,8)$ & $39,9(21,6)$ & 138,5 \\
Somatización & $0,73(, 69)$ & $0,9(0,8)$ & $0,7(0,7)$ & 161,5 \\
Obsesión-compulsión & $0,96(0,80)$ & $1,3(0,9)$ & $0,9(0,7)$ & 138 \\
Sensibilidad interpersonal & $1,02(0,84)$ & $1,2(0,7)$ & $1,01(0,8)$ & 129 \\
Depresión & $1,18(0,90)$ & $1,3(0,9)$ & $1,2(0,9)$ & 165 \\
Ansiedad & $0,94(1,07)$ & $1,01(0,7)$ & $0,9(1,2)$ & 149 \\
Hostilidad & $0,66(0,74)$ & $0,5(0,4)$ & $0,7(0,8)$ & 178,5 \\
Ansiedad fóbica & $0,56(0,95)$ & $0,6(0,6)$ & $0,5(1,1)$ & 125 \\
Ideación paranoide & $0,89(0,81)$ & $1,2(0,8)$ & $0,8(0,8)$ & 117 \\
Psicoticismo & $0,73(0,68)$ & $0,8(0,7)$ & $0,7(0,7)$ & 161 \\
\hline
\end{tabular}


vención clínica con alcohólicos no contemplan, en su mayor parte, la evaluación del juego patológico. Sin embargo, los resultados de este estudio muestran la necesidad de tener en cuenta los problemas de juego, tanto en la evaluación clínica de la adicción al alcohol, como en el desarrollo de programas específicos de tratamiento. En caso contrario, el problema puede permanecer oculto, o solapado en la dependencia alcohólica, y dificultar enormemente la intervención terapéutica.

Por otra parte, no se debe olvidar el riesgo existente en el tratamiento de las conductas adictivas de desarrollar una dependencia sustitutiva. En estos casos, el tratamiento del alcoholismo puede agravar los problemas de juego patológico presentados por el paciente o, una vez conseguida la abstinencia, precipitar una recaída en el consumo de alcohol. En el estudio de Fernández-Montalvo, Echeburúa y Báez (1999), por ejemplo, el consumo de alcohol constituía una de las variables precipitantes más importantes de las recaídas en el tratamiento de la ludopatía. Por todo ello, algunos autores han propuesto, incluso, la posibilidad de llevar a cabo en este tipo de pacientes un tratamiento conjunto de la dependencia alcohólica y del juego patológico, con el objetivo de prevenir el desarrollo de una eventual dependencia sustitutiva (Daghestani et al. 1996; Rodríguez-Martos, 1989).

Por otra parte, la comparación entre los alcohólicos con un problema asociado de juego patológico y los alcohólicos sin problemas de ludopatía muestra la existencia de diferencias significativas en las características relacionadas con el consumo de alcohol. Más en concreto, los alcohólicos con problemas de juego patológico presentan una mayor puntuación en el MALT, una mayor frecuencia de consumo y una mayor probabilidad de presentar otras dependencias asociadas.
Ello refleja la existencia de una mayor gravedad de la adicción (en términos de consumo y de polidependencia) en los pacientes alcohólicos aquejados también de juego patológico. Probablemente, el solapamiento habitual del ambiente social de los alcohólicos y de los jugadores (bares, pubs, etc.), influya en este sentido, ya que en el mismo local de juego se puede consumir alcohol, y viceversa.

No obstante, una limitación de este estudio es que los resultados presentados se han obtenido con una muestra relativamente pequeña (50 alcohólicos). Por ello, sería conveniente replicar esta investigación con una muestra de alcohólicos más amplia. Asimismo, los datos de esta investigación no permiten conocer cuál es la relación temporal entre ambas adicciones. En el estudio de Rodríguez-Martos (1989), por ejemplo, el $57 \%$ de los sujetos consideraba que el problema de alcoholismo era anterior al problema de juego patológico. Este hecho constituye un aspecto interesante para la investigación futura.

\section{REFERENCIAS}

Alonso-Fernández, F. (1996). Las otras drogas. Madrid: Temas de Hoy

American Psychiatric Association (1980). Diagnostic and statistical manual of mental disorders ( $3^{\text {rd }}$ ed. rev.). Washington, D.C.: APA.

American Psychiatric Association (2000). Diagnostic and statistical manual of mental disorders ( $4^{\text {th }} e d$. rev.). Washington, D.C.: APA.

Becoña, E. (1993). El juego compulsivo en la comunidad autónoma gallega. Santiago de Compostela: Xunta de Galicia.

Becoña, E. (1996). Prevalence surveys of problem and pathological gambling in Europe: The cases of Germany, Holland and Spain. Journal of Gambling Studies, 12, 179-192.

Bland, R.C., Newman, S.C., Orn, H. y Stebelsky, G. (1993). Epidemiology of patho- 
logical gambling en Edmonton. Canadian Journal of Psychiatry, 38, 108-112.

Casas, M. (1994). Psicopatología y alcoholismo. Barcelona. Citrán.

Ciarrocchi, J.M. y Richardson, J. (1989). Profile of compulsive gamblers in treatment. Update and comparisons. Journal of Gambling Behavior, 5, 53-65.

Daghestani, A.N., Elenz, E. y Crayton, J.W. (1996). Pathological gambling in hospitalized substance abusing veterans. Journal of Clinical Psychiatry, 57, 360-363.

Dell, L.J., Ruzicka, M.F. y Palisi, A.T. (1981). Personality and other factors associated with the gambling addiction. International Journal of Addiction, 16, 149-156.

Derogatis, L.R. (1992). The SCL-90-R. Baltimore: Clinical Psychometric Research.

Echeburúa, E. (1999). ¿Adicciones sin drogas? Las nuevas adicciones. Bilbao: Desclée de Brouwer.

Echeburúa, E. (2001). Abuso de alcohol. Madrid: Síntesis.

Echeburúa, E., Báez, C., Fernández-Montalvo, J. y Páez, D. (1994). Cuestionario de Juego Patológico de South Oaks (SOGS): validación española. Análisis y Modificación de Conducta, 20, 769-791.

Fernández-Montalvo, J., Echeburúa, E. y Báez, C. (1999). Las recaídas en el juego patológico: un estudio de las situaciones precipitantes. Revista de Psicopatología y Psicología Clínica, 4, 115-121.

Fernández-Montalvo, J. y Landa, N. (2003). Comorbilidad del alcoholismo con los trastornos de personalidad. Clínica y Salud, 14, 27-41.

Fernández-Montalvo, J., Landa, N., LópezGoñi, J.J., Lorea, I. y Zarzuela, A. (2002). Trastornos de personalidad en alcohólicos: un estudio descriptivo. Revista de Psicopatología y Psicología Clínica, 7, 217-225.

Feuerlein, W., Ringer, C.H. y Kufner, K.A. (1977). Diagnose des alkoholismus: der Müncher Alkoholismus Test (MALT). München Med Woschenchr, 119, 1275-1282.

González de Rivera, J.L. (2002). Versión española del SCL-90-R. Madrid: TEA.

Kausch, O. (2003). Patterns of substance abuse among treatment-seeking pathological gamblers. Journal of Substance Abuse Treatment, 25, 263-270.
Ladouceur, R. (1991). Prevalence estimates of pathological gamblers in Québec, Canada. Canadian Journal of Psychiatry, 36, 732734.

Ledgerwood, D.M. y Downey, K.K. (2002). Relationship between problem gambling and substance use in a methadone maintenance population. Addictive Behaviors, 27, 483-491.

Lejoyeux, M., Feuche, N., Loi, S., Solomon, J. y Ades, J. (1999). Study of impulse control disorders among alcohol dependent patients. Journal of Clinical Psychiatry, 60, 302-305.

Lesieur, H.R. y Blume, S.B. (1987). The South Oaks Gambling Screen (SOGS): A new instrument for the identification of pathological gamblers. American Journal of Psychiatry, 144, 1184-1188.

Lesieur, H.R., Blume, S.B. y Zoppa, R.M. (1986). Alcoholism, drug abuse and gambling. Alcoholism: Clinical and Experimental Research, 10, 33-38.

Martínez-Pina, A., Guirao de Parga, J.L., Fusté, R., Serrat, X., Martín, M. y Moreno, V. (1991). The Catalonia Survey: personality and intelligence structure in a sample of compulsive gamblers. Journal of Gambling Studies, 7, 275-299.

Ramírez, L.F., McCormick, R.A., Russo, A.M. y Taber, J.L. (1983). Patterns of substance abuse in pathological gamblers undergoing treatment. Addictive Behaviors, 8 , 425-428.

Rodríguez-Martos, A. (1989). Estudio piloto estimativo de la prevalencia de juego patológico entre los pacientes alcohólicos que acuden al Programa DROSS. Revista Española de Drogodependencias, 14, 265-275.

Rodríguez-Martos, A. y Suárez, R. (1984). MALT (Munchner Alkoholismus Test). Validación de la versión española de este test para el diagnóstico de alcoholismo. Revista de Psiquiatría y Psicología Médica, 16, 421-432.

Sellman, J.D., Adamson, S., Robertson, P., Sullivan, S. y Coverdale, J. (2002). Gambling in mild-moderate alcohol-dependent outpatients. Substance Use and Misuse, 37, 199-213.

Specker, S.M., Carlson, G.A., Edmonson, K.M., Johnson, P.E. y Marcotte, M. (1996). 
Psychopathology in pathological gamblers seeking treatment. Journal of Gambling Studies, 12, 67-81.

Spunt, B., Dupont, I., Lesieur, H., Liberty, H.J. y Hunt, D. (1998). Pathological gambling and substance misuse: a review of the literature. Substance Use and Misuse, 33, 2535-2560.

Toneatto, T. y Brennan, J. (2002). Pathological gambling in treatment-seeking substance abusers. Addictive Behaviors, 27, 465469.

Toneatto, T., Ferguson, D. y Brennan, J. (2003). Effect of a New Casino on problem gambling in treatment-seeking substance abusers. Canadian Journal of Psychiatry, 48, 40-44.
Volberg, R.A. y Abbott, M.W. (1994). Lifetime prevalence estimates of pathological gambling in New Zealand. International Journal of Epidemiology, 23, 976-983.

Volberg, R.A. y Steadman, H.J. (1988). Refining prevalence estimates of pathological gambling. American Journal of Psychiatry, 145, 502-505.

Volberg, R.A. y Steadman, H.J. (1989). Prevalence estimates of pathological gambling in New Jersey and Maryland. American Journal of Psychiatry, 146, 1618-1619.

Welte, J.W., Barnes, G.M., Wieczorek, W.F. y Tidwell, M.C. (2004). Simultaneous drinking and gambling: a risk factor for pathological gambling. Substance Use and Misuse, 39, 1405-1422. 